
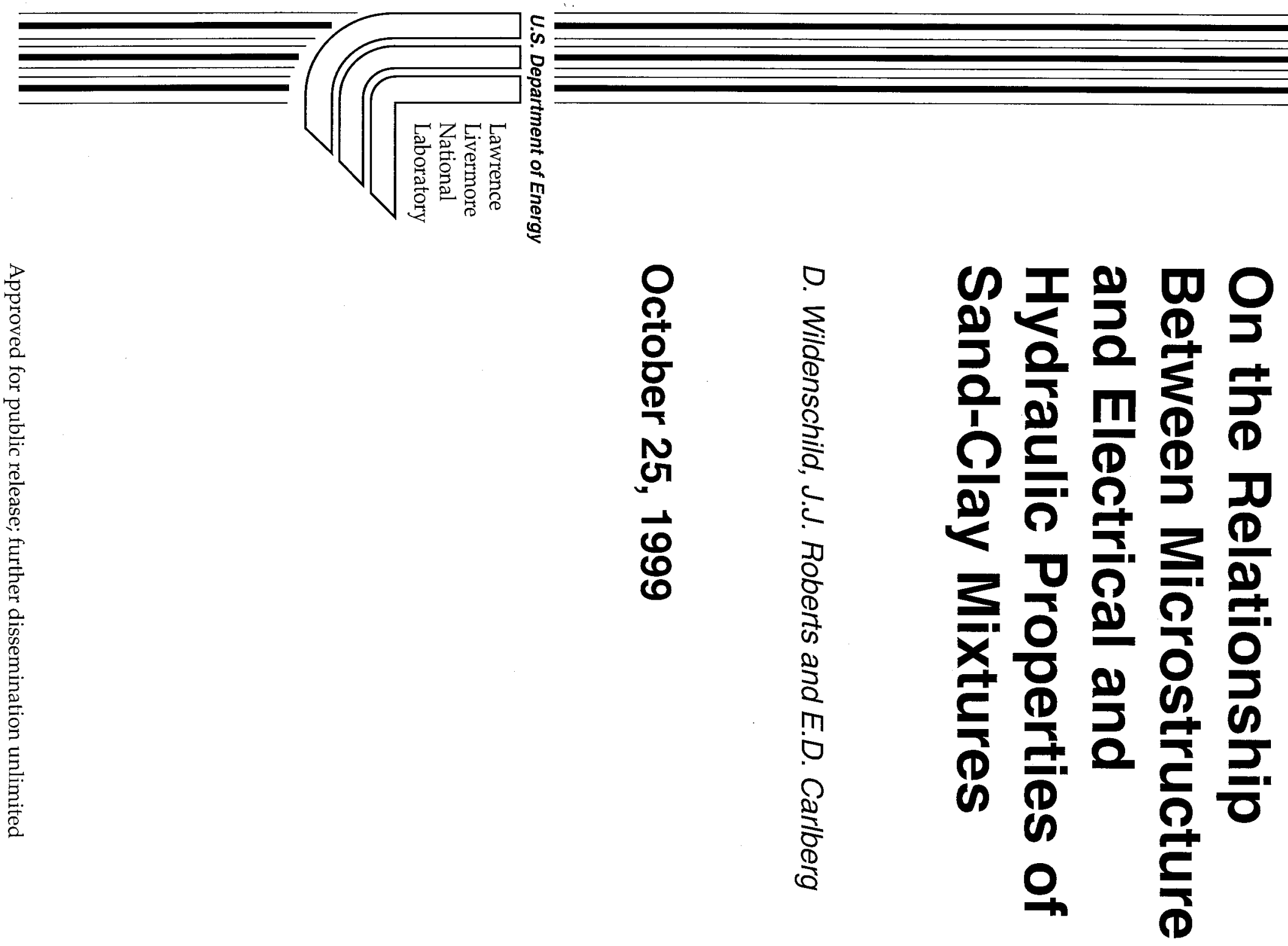


\section{DISCLAIMER}

This document was prepared as an account of work sponsored by an agency of the United States Government. Neither the United States Government nor the University of California nor any of their employees, makes any warranty, express or implied, or assumes any legal liability or responsibility for the accuracy, completeness, or usefulness of any information, apparatus, product, or process disclosed, or represents that its use would not infringe privately owned rights. Reference herein to any specific commercial product, process, or service by trade name, trademark, manufacturer, or otherwise, does not necessarily constitute or imply its endorsement, recommendation, or favoring by the United States Government or the University of California. The views and opinions of authors expressed herein do not necessarily state or reflect those of the United States Government or the University of California, and shall not be used for advertising or product endorsement purposes.

Work performed under the auspices of the U. S. Department of Energy by the University of California Lawrence Livermore National Laboratory under Contract W-7405-Eng-48.

This report has been reproduced directly from the best available copy.

Available to DOE and DOE contractors from the

Office of Scientific and Technical Information

P.O. Box 62, Oak Ridge, TN 37831

Prices available from (423) 576-8401

http:/ / apollo.osti.gov/bridge/

Available to the public from the National Technical Information Service

U.S. Department of Commerce 5285 Port Royal Rd., Springfield, VA 22161

http://www.ntis.gov/

OR

Lawrence Livermore National Laboratory

Technical Information Department's Digital Library

http://www.llnl.gov/tid/Library.html 


\title{
On the Relationship Between Microstructure and Electrical and Hydraulic Properties of Sand-Clay Mixtures
}

\author{
Dorthe Wildenschild, Jeffery J. Roberts, and Eric D. Carlberg \\ Experimental Geophysics Group, Lawrence Livermore National Laboratory \\ P.O. Box 808, L-202, Livermore, CA 94551, 925-422-0257, wildenschild1@îllnl.gov
}

\begin{abstract}
A series of laboratory experiments were performed on saturated sand-clay mixtures, including electrical properties, permeability and porosity. Different mixtures and configurations of quartz sand and 0 to $10 \% \mathrm{Na-montmorillonite} \mathrm{clay} \mathrm{saturated} \mathrm{with} \mathrm{solutions} \mathrm{of} \mathrm{CaCl}_{2}$ and deionized water were investigated. The electrical properties were dependent on clay content, fluid conductivity, and microstructure in a complex fashion. Two main regions of conduction exist: a region dominated by surface conduction and a region where the ionic strength of the saturating fluid controlled conduction. For low fluid conductivities, the sample geometry was found to greatly affect the magnitude of the surface conductance. The influence of the microstructural properties on the electrical properties was quantified by estimating formation factors, A-parameters, and surface conductances. We suggest that high and low bounds on the expected surface and bulk conductance of natural systems can be derived from the measurements on these artificial configurations.
\end{abstract}

\section{Introduction}

Electrical measurements are a useful, nondestructive tool for characterizing porous rocks and soils. Conduction of elcctricity through porous media occurs by two mechanisms. The primary mode of conduction is by movement of ions through the bulk-saturating electrolyte. Conduction also takes place as adsorbed ions move along the surfaces of pores and cracks. Thus, the 
conductivity of a porous medium is related to microstructural properties such as porosity, pore geometry, and surface morphology of the mineral grains lining the pores as well as to the dielectric properties of the mineral grains and pore fluid. Electrical conduction is also a function of the prevailing fluid saturation. For brine-saturated porous materials, Archie (1942) proposed the following relationship between bulk and fluid conductivities:

$$
\sigma_{b u l k}=\sigma_{\text {fluid }} a \varphi^{m}
$$

where $\sigma_{b u l k}$ is the electrical conductivity of the porous medium, $\sigma_{f t u i d}$ is the electrical conductivity of the saturating fluid, $\varphi$ is the porosity, and $a$ and $m$ are parameters that are supposed to be constant for a certain type of rock. The ratio $\sigma_{\text {fluid }}: \sigma_{b u l k}$ is the formation factor $F$. Equation 1 assumes that the contribution of surface conduction to bulk conduction is negligible. If that is not the case, it has been found in theoretical (Johnson et al., 1986) and experimental work (Waxman and Smits, 1968; Sen et al., 1988; and Sen and Goode, 1992) that Archie's law has to be modified to include a surface-conduction term. Johnson et al. (1986) defined a length parameter $\Lambda$, which is a weighted volume-to-surface-area ratio (a measure of the dynamically interconnected pore size) defined as

$$
\frac{\Lambda}{2}=\frac{\int|E(r)|^{2} d V_{p}}{\int|E(r)|^{2} d S}
$$

$\mathrm{E}(\mathrm{r})$ is the electric potential field at point $r, V_{p}$ is the pore volume, and $S$ is the surface area (i.e., the pore-solid interface). $A$ has units of length and is a parameter characteristic of the geometry of the porous medium. It is therefore transferable from one experiment to another for a specific medium. This relation is valid for materials where the (insulating) grains are coated with appreciable amounts of clay minerals (Johnson et al., 1986). When dry clays are saturated with brincs, the counter ions, which usually balance out charged impurities by bonding to their 
external surfaces, hydrate and become mobile within a layer. Surface conduction due to these mobile counter ions then acts in parallel with the ionic conduction associated with the brine. For relatively high-salinity pore fluids, a linear relationship was derived (Johnson et al., 1986):

$$
\sigma_{b u l k}=\frac{1}{F}\left(\sigma_{\text {fluid }}+\frac{2 \Sigma_{s}}{\Lambda}\right)
$$

where $\Sigma_{s}$ is the surface conductivity. When $\sigma_{\text {fluid }}$ and $\sigma_{\text {bulk }}$ are plotted on a linear scale, the slope equals the reciprocal of the formation factor $F$, and the intercept with the ordinate axis $b=$ $2 \Sigma_{s} / F \Lambda$. As mentioned previously, several investigators have demonstrated the presence of the intercept for clay-bearing materials. The phenomenon was particularly well explained by Glover et al. (1994). The objective of the present study was to investigate the influence of various microstructural properties (such as clay content and configuration) on the electrical properties represented by varying formation factors, $\Lambda$ parameters, and surface conductances $\Sigma_{s}$. We used a number of different configurations containing 0 to $10 \%$ clay: dispersed mixtures, discrete clay clusters, and arrangements of distinct layers of clay in the sand matrix. Solutions of $\mathrm{CaCl}_{2}$ ranging from $0.0005 \mathrm{~N}$ to $0.75 \mathrm{~N}(0.05$ to $64 \mathrm{mS} / \mathrm{cm})$ and deionized water were used as

saturating fluids. The range of fluid conductivities was chosen to include the range of conductivities commonly found in natural groundwater $(0.5-1.0 \mathrm{mS} / \mathrm{cm})$.

\section{Experimental Details}

\subsection{Sample Preparation and Characterization}

Ottawa sand from U.S. Silica (F-50) and Na-montmorillonite (Wyoming bentonite) were used to build the unconsolidated samples. The Ottawa sand is a pure silica sand with grain sizes between 74 and 420 microns, a median diameter $\left(d_{50}\right)$ of 273 microns, and a particle density of $2.66 \mathrm{~g} / \mathrm{cm}^{3}$. The samples were packed in 2-in.-diameter heat-shrink Teflon tubing with sintered 
Hasteloy frits at both ends providing support and allowing fluid flow. Sample height was 1.5 in., and $\mathrm{AgCl}$-coated silver wire electrodes were positioned in the casing $0.25 \mathrm{in}$. from either end of the sample using additional heat-shrink material as spacing. The electrodes were electrochemically coated with $\mathrm{AgCl}$. Once the sand-clay mixture was packed between frits, the tubing was heated and subsequently shrank to provide semirigid support for the unconsolidated sample. We used samples of varying clay content and configuration. All percentage mixtures of sand and clay were prepared by weight $(1 \%, 3 \%$, or $10 \%)$. The dispersed samples were packed from dry mixed sand and clay. The samples having cluster configurations were mixed from dry sand and predetermined amounts of dried bits of clay. The materials were well mixed before the mixture was packed in the sample holder. X-ray tomography on one such sample showed that the clusters were well distributed in the sample. The clusters varied in size and shape but were $\sim 0.5$ $-1.0 \mathrm{~cm}$ diameter when dry.

The samples were saturated with saline fluids of varying concentration, ranging from 0.0005 $\mathrm{N}$ to $0.75 \mathrm{~N}$ of $\mathrm{CaCl}_{2}(0.05$ to $64 \mathrm{mS} / \mathrm{cm})$. Mcasurements were also made using de-ionized water.

\subsection{Apparatus and Measurement Procedures}

The experimental setup consists of four major components: the sample, the sample holder with pressure controls, the electrical measurement system, and the fluid-flow system (Figure 1). The prepared soil sample was placed in a Hassler sleeve, which controlled the pore pressure in the sample during the experiment. The Hassler sleeve was held in place by end-load and in-load pressures using air-actuated cylinders (Bimba), ensuring that the sample was hydraulically sealed in the holder (i.e., sidewall flow was avoided) and also provided for simulating shallow burial.

Various fluids were pumped through the samples and the electrical properties were measured using an HP 4284A LCR-meter and employing the four-electrode method as outlined by Olhoeft 
(1985). Electrical measurements were made at $1 \mathrm{kHz}$ using the $\mathrm{HP} 4284 \mathrm{~A}$ while the sample equilibrated to a new saturating solution.

Following successful measurement of the electrical properties, we used a constant flow technique to measure hydraulic permeability. A Harvard syringe pump provided constant flowrates through the sample, and pressure transducers were used to measure to hydraulic pressure gradient across the sample. Darcy's Law was then applied to obtain the hydraulic conductivity $K$ and permeability $k$ :

$$
\begin{gathered}
K=-\frac{Q}{A} \frac{1}{d H / d X} \\
k=K \frac{\mu}{\rho_{w} g}
\end{gathered}
$$

where $Q$ is the volumetric flux, $A$ is cross-sectional area, $d H / d X$ is the total soil-water potential gradient between the two tensiometers, $\mu$ is the dynamic viscosity, $\rho_{w}$ is the density of the fluid, and $g$ is gravitational acceleration. All hydraulic conductivities/permeabilities were measured using deionized water.

\section{Results and Discussion}

\subsection{Hydraulic Permeability Measurements}

We performed several tests to confirm that permeability was independent of fluid salinity. Three consecutive measurements were performed on two samples containing 3\% Namontmorillonite. On one sample, we first measured the permeability using deionized water, then measured it using a $0.1 \mathrm{~N} \mathrm{CaCl}_{2}$ solution, and finally repeated the measurement using deionized water. The second sample was subject to permeability measurements with $0.1 \mathrm{~N} \mathrm{CaCl}_{2}$, 
deionized water, and $0.1 \mathrm{~N} \mathrm{CaCl}_{2}$, in that order. The measured permeabilities for these two test samples are listed in Table 1.

Table 1. Permeabilities measured consecutively using deionized water and a $0.1 \mathrm{~N} \mathrm{CaCl}_{2}$ solution on two test samples containing $3 \%$ clay.

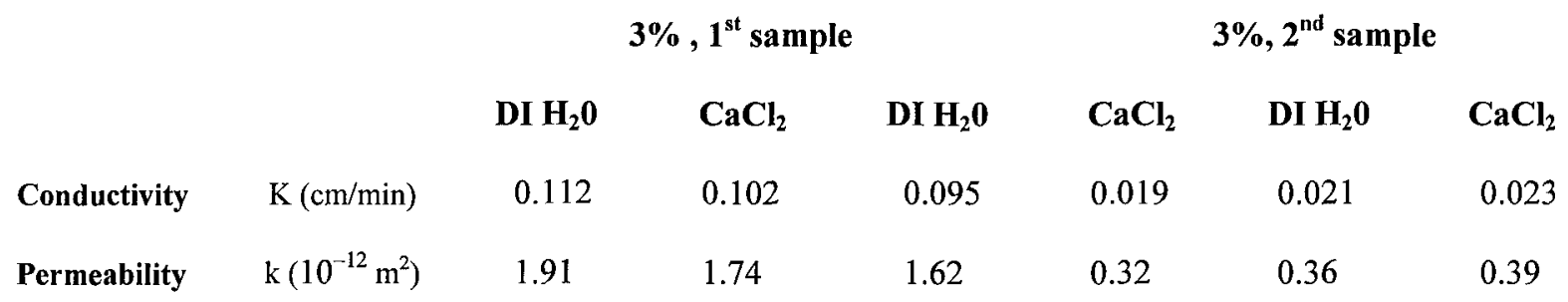

We found only minor variation among permeabilities measured using $\mathrm{CaCl}_{2}$ and using deionized water and no consistent change of permeability with time or total volume of water flowed. The standard deviations for the three measurements, made on the two different samples, were $0.15 \cdot 10^{-12} \mathrm{~m}^{2}$ and $0.03 \cdot 10^{-12} \mathrm{~m}^{2}$, respectively. The difference in permeability of the two samples, however, was significant: a factor of approximately 5 . We attribute this to variation in packing and compaction and, thus, to variation in bulk density and porosity of the individual samples.

Permeabilities measured on all the sand-clay samples are listed in Table 2. These permeabilities varied within two orders of magnitude. The highest was measured for the clean sand sample $\left(6.1 \cdot 10^{-12} \mathrm{~m}^{2}\right)$, and the lowest was found for the $10 \%$-dispersed clay sample $\left(0.048 \cdot 10^{-12} \mathrm{~m}^{2}\right)$. The two other samples containing $10 \%$ clay (non-dispersed) had permeabilities similar to that of the clean sand sample. Apparently the clay content was not impeding fluid flow significantly when it was configured as clusters or as a clay layer parallel to the direction of flow. 
Table 2. Hydraulic and electrical properties measured on the various samples

\begin{tabular}{|c|c|c|c|c|c|c|c|c|}
\hline & & $\begin{array}{c}\text { F50 } \\
\text { clean } \\
\text { sand }\end{array}$ & $\begin{array}{l}1 \% \text { clay } \\
\text { dispersed }\end{array}$ & $\begin{array}{l}3 \% \text { clay } \\
\text { dispersed }\end{array}$ & $\begin{array}{l}3 \% \text { clay } \\
\text { clusters }\end{array}$ & $\begin{array}{l}10 \% \text { clay } \\
\text { dispersed }\end{array}$ & $\begin{array}{c}10 \% \text { clay } \\
\text { clusters }\end{array}$ & $\begin{array}{c}10 \% \text { clay } \\
\text { parallel } \\
\text { layer }\end{array}$ \\
\hline $\begin{array}{c}\text { Conductivity } \\
\text { (DI) }\end{array}$ & $\begin{array}{c}\mathrm{K} \\
(\mathrm{cm} / \mathrm{min})\end{array}$ & 0.360 & & 0.097 & & 0.003 & 0.156 & 0.228 \\
\hline $\begin{array}{l}\text { Permeability } \\
\text { (DI) }\end{array}$ & $\left(10^{-12} \mathrm{~m}^{2}\right)$ & 6.14 & & 1.65 & & 0.05 & 2.73 & 3.92 \\
\hline Porosity & & 0.344 & & 0.365 & & 0.357 & 0.306 & 0.383 \\
\hline Slope & $\mathrm{m}$ & 0.206 & & 0.197 & & 0.076 & 0.225 & 0.196 \\
\hline Intercept & $\mathrm{b}(\mathrm{S} / \mathrm{m})$ & 0.0054 & & 0.0188 & & 0.0576 & 0.0167 & 0.0757 \\
\hline $\begin{array}{l}\text { Formation } \\
\text { factor }\end{array}$ & $\mathrm{F}(=1 / \mathrm{m})$ & 4.9 & & 5.1 & & 13.2 & 4.4 & 5.1 \\
\hline$\Lambda$ parameter & $\Lambda(\mu \mathrm{m})$ & 15.4 & & 8.2 & & 2.3 & 9.8 & 12.7 \\
\hline $\begin{array}{c}\text { Surface } \\
\text { conductance }\end{array}$ & $\Sigma_{\mathrm{S}}(\mu \mathrm{S})$ & 0.20 & & 0.39 & & 0.85 & 0.36 & 2.45 \\
\hline $\begin{array}{c}\text { Archie's } \\
\text { exponent }\end{array}$ & & 1.48 & & 1.61 & & 2.51 & 1.26 & 1.70 \\
\hline
\end{tabular}

\subsection{Elcctrical Measurements}

The single-frequency measurements are reported at $1 \mathrm{kHz}$. Measurements at this frequency generally have a phase angle near zero and thus mostly contain the resistive component. Measurements were made using $\mathrm{CaCl}_{2}$ as the saturating fluid, except for a few measurements performed using $\mathrm{NaCl}$ solutions. For two solutions $\left(\mathrm{CaCl}_{2}, \mathrm{NaCl}\right)$ of the same fluid conductivity, we found practically identical values of the bulk sample conductivity. The electrical measurements are illustrated in Figures 2 and 3. Figure 2 is a $\log -\log$ plot of fluid conductivity versus bulk sample conductivity for the various samples; Figure 3 is the analogous linear plot with fitted straight lines. The slopes and intercepts of these fitted lines are listed in Table 2.

There are two different regimes visible in Figure 2 . At high fluid concentrations $(>1 \mathrm{~S} / \mathrm{m})$, the logarithm of the bulk conductivity has a linear dependence on the logarithm of the fluid conductivity, and the slope of the line is approximately unity. Movement of ions through the bulk-fluid phase is the dominant mechanism of conduction in this regime. However, at low fluid concentrations $(<1 \mathrm{~S} / \mathrm{m}$ ), the bulk conductivity is no longer solely dependent on the fluid 
conductivity. In this regime, surface conductance due to mobile ions in the electrical double layer of the clay becomes more important. In the lower fluid concentration regime, the curves tend to a constant, which represents the value of the surface conductivity. These mechanisms are readily demonstrated in Figures 2 and 3. The clean-sand sample has a straight-line dependence of $\sigma_{b u l k}$ on $\sigma_{\text {fluid }}$ and a low intercept of $0.0054 \mathrm{~S} / \mathrm{m}$ (i.e., a low surface conductance). As the clay content increases, we see an increasing effect of surface conductance on the value of the intercept. With a $3 \%$-dispersed clay content, the intercept is at $0.0188 \mathrm{~S} / \mathrm{m}$, increasing to $0.0576 \mathrm{~S} / \mathrm{m}$ for $10 \%$ dispersed clay. A significant change is seen when the configuration of the clay is altered; $10 \%$ clay configured as clusters gives a lower intercept $(0.0167 \mathrm{~S} / \mathrm{m})$ (i.e., lower surface conductivity) than does a 3\%-dispersed configuration, and a 10\%-layered configuration results in the highest intercept $(0.0757 \mathrm{~S} / \mathrm{m})$ overall. These variations in the contribution of the surface conductance to the bulk conductance are discussed in Section 3.3 .

\subsection{Electrical Parameters}

We estimate various electrical parameters based on the theory of Johnson et al. (1986). From the slope and intercept of the curves in Figure 3, we calculate the formation factors as the reciprocal of the slope. For most of the samples, the formation factors are $\sim 5$, except for the 10\%-dispersed sample, which has a formation factor of $\sim 13$.

Under certain conditions, the $\Lambda$-parameter can be correlated with the hydraulic permeability $k$ and the formation factor $F$ (Johnson et al, 1986; Kostek et al., 1992):

$$
\Lambda=\sqrt{k 8 F}
$$

We use this equation to calculate the $\Lambda$-parameters listed in Table 2 . A high $\Lambda$-parameter of 15.4 $\mu \mathrm{m}$ is found for the clean-sand sample, while the $10 \%$-dispersed clay sample has a $\Lambda$-parameter 
of $2.3 \mu \mathrm{m}$. We expected a higher $\Lambda$-parameter for a sample with a lower surface area (and thus a lower clay content) and vice versa because $\Lambda \approx V_{p} / S$.

Because the sample volume is practically identical for all samples, the $\Lambda$-parameter becomes approximately proportional to $1 / S$. The surface conductances listed in Table 2 are calculated from the intercept $b$ as (see Equation 3 for derivation):

$$
\Sigma_{s}=\frac{b \Lambda F}{2}
$$

The surface conductances vary between $0.20 \mu \mathrm{S}$ for the clean sand sample and $2.45 \mu \mathrm{S}$ for the sample containing 10\%-layered clay. In comparison, Nettelblad et al. (1995) found surface conductances in the range $0.05-0.15 \mu \mathrm{S}$ for artificially made, clay-free sandstones. The estimated values of $\Sigma_{s}$ follow the trends observed in Figure 2. An increasing surface conductance with increasing clay content is observed for the dispersed samples; once the configuration changes, that is no longer the case. The 10\%-clustered sample has the lowest surface conductance (of the clay-bearing samples), $\Sigma_{s}=0.36 \mu \mathrm{m}$, even lower than the sample containing $3 \%$-dispersed clay; the $10 \%$-layered sample, on the other hand, has by far the highest surface conductance. This is intuitively acceptable because the artificially constructed layered-clay configuration provides a preferential path for ion transfer between the frits, whereas clay in clusters is rarely interconnected and has a comparatively low surface area, even relative to the dispersed sample. The measurements on the two extreme configurations of the clustered and the parallel samples may provide bounds on the expected bulk conductivity of clay-bearing sandy soils and thereby help predict conditions in natural systems. The more "natural" configuration of the $10 \%$ dispersed sample falls nicely within these bounds with a surface conductance of $0.85 \mu \mathrm{m}$.

\section{Conclusion}


We have investigated the influence of various microstructural properties on the electrical properties represented by varying formation factors, $\Lambda$-parameters, and surface conductances. We found fairly similar formation factors and 1 -parameters for all the clay-bearing samples, except for the sample containing $10 \%$ dispersed clay. Based on these parameters only, one could expect that certain clay-related physical or chemical processes could be neglected for clay contents of less than $10 \%$. However, the surface conductance varied significantly with clay content and configuration. An increasing surface conduction was observed as the samples contained increasingly larger amounts of dispersed clay. The configurations of the clay also played a major role, with the clustered and parallel configurations having the lowest and highest surface conductances, respectively. Apparent high and low bounds on the expected surface and bulk conductance in a natural system may be derived from the measurements on these more artificial configurations and may thereby provide valuable information for inverse modeling of conditions in natural systems.

\section{Acknowledgments}

The authors are very thankful to C. Boro, B. Ralph, P. Berge, B. Bonner, J. Berryman, D. Rikard, and R. Perry of Lawrence Livermore National Laboratory and to D. Johnson of Solartron, Inc.

This work was performed under the auspices of the U.S. Department of Energy by Lawrence Livermore National Laboratory under Contract Number W-7405-ENG-48 and supported specifically by the Environmental Management Science Program of the Office of Environmental Management and the Office of Energy Research. 


\section{References}

Archie, G.E., 1942, The electrical resistivity $\log$ as an aid in determining some reservoir characteristics: Trans. AIME, 146, 54-62.

Glover, P.W.J., P.G. Meredith, P.R. Sammonds, and S.A.F. Murrell, 1994, Ionic surface electrical conductivity in sandstone: J. Geophys. Res., 99, 21635-21650.

Johnson, D.L., J. Koplik, and L.M. Schwartz, 1986, New pore-size parametcr characterizing transport in porous media: Phys. Rev. Lett, 57, 2564-2567.

Kostek, S., L.M. Schwartz, and D.L. Johnson, 1992, Fluid permeability in porous media: Comparison of electrical estimates with hydrodynamical calculations: Phys. Rev. B, 45,186-195.

Nettelblad, B., B. Åhlén, G.A. Niklasson, and R.M. Holt, 1995, Approximate determination of surface conductivity in porous media: J. Phys. D: Appl. Phys., 28, 2037-2045.

Olhoeft, G.R., 1985, Low-frequency electrical properties: Geophysics, 50, 2492-2503.

Sen, P.N., P.A. Goode, and A. Sibbit, 1988, Electrical conduction in clay bearing sandstones at low and high salinities: J. Appl. Phys., 63, 4832-4840.

Sen, P.N. and P.A. Goode, 1992, Influence of temperature on electrical conductivity on shaly sands: Geophysics, 57, 89-96.

Waxman, M.H. and L.J.M. Smits, 1968, Electrical conductivities in oil-bearing shaly sands: Soc. Pet. Eng. J., 8, 107-122. 


\section{Captions}

Figure 1. Experimental setup.

Figure 2. Log-log plot of $\rho_{\text {bulk }}$ Vs. $\rho_{\text {fluid. }}$

Figure 3. Linear plot of $\rho_{b u l k}$ vs $\rho_{f l u i d}$ 

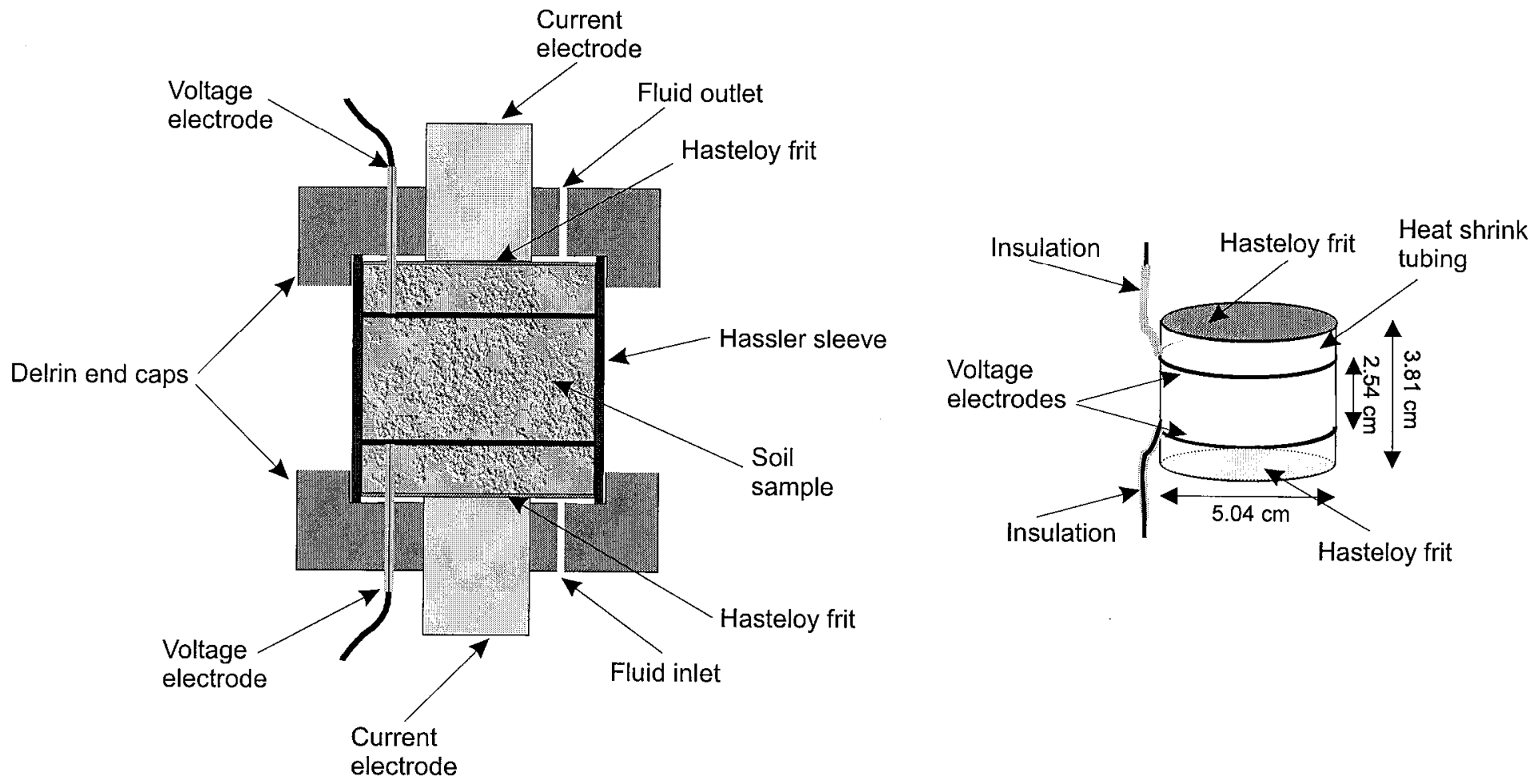


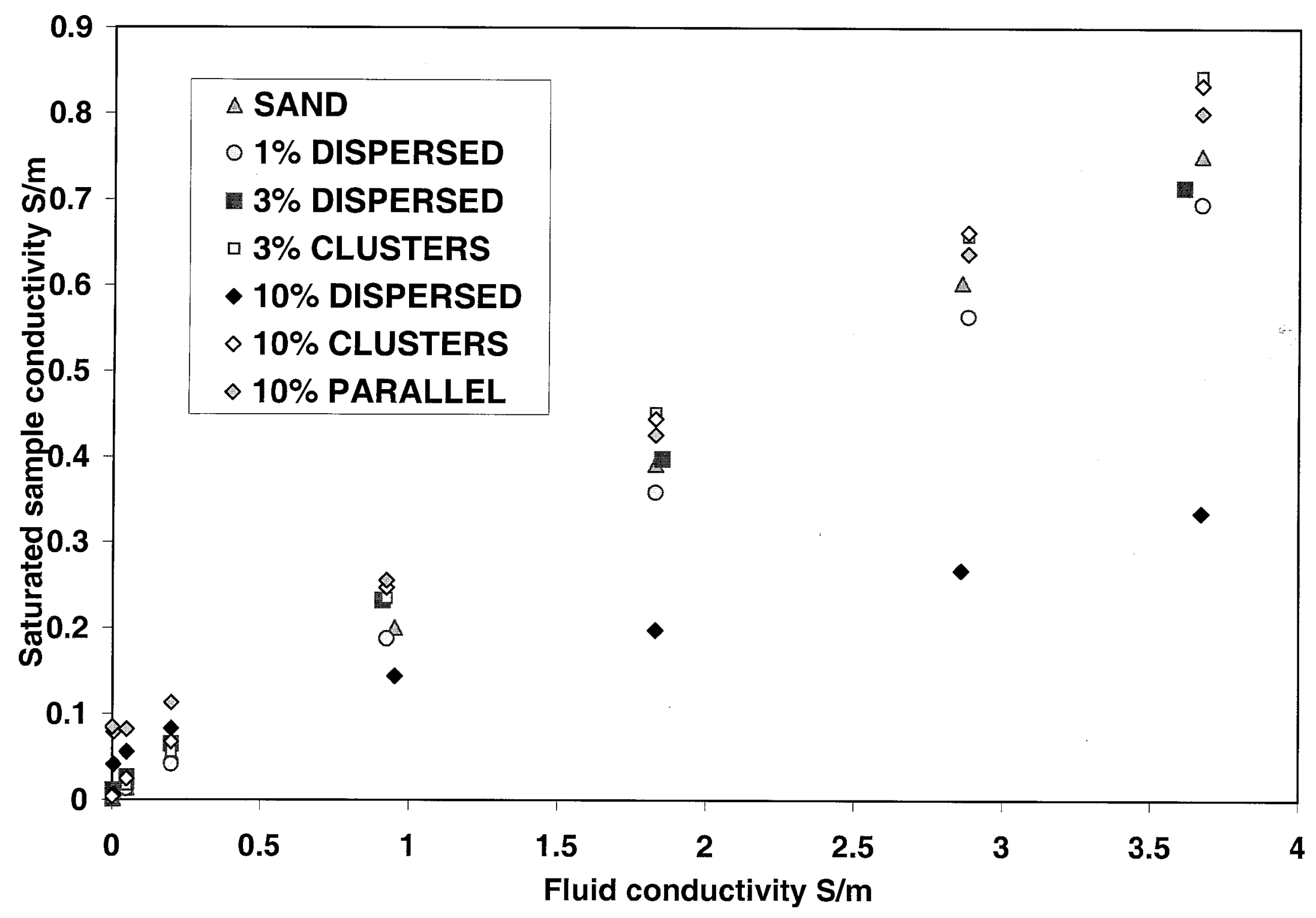




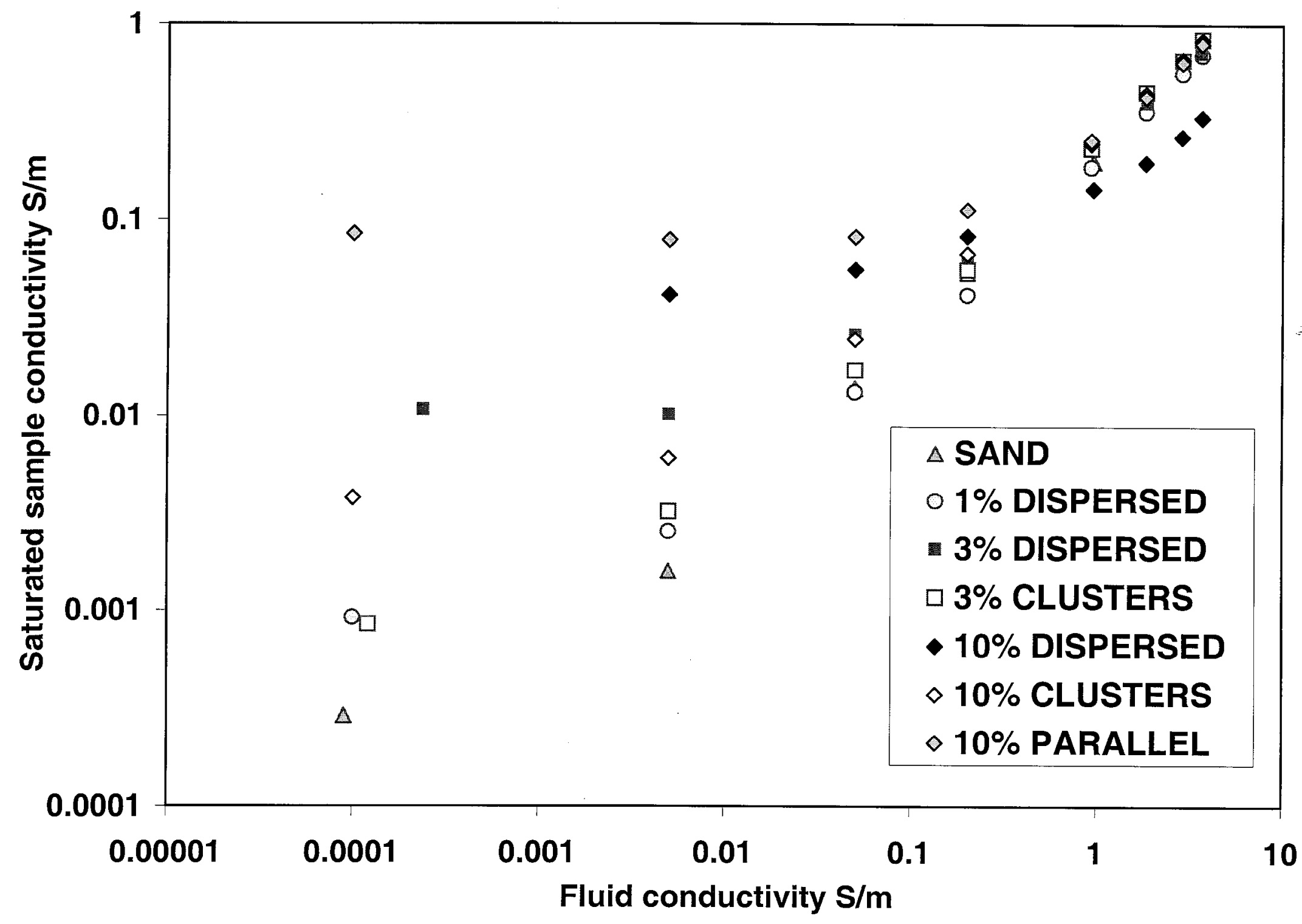

Fig. 3 